\title{
Spatial Stroop and spatial orienting: the role of onset versus offset cues
}

\author{
Chunming Luo $\cdot$ Juan Lupiáñez $\cdot$ \\ Xiaolan Fu $\cdot$ Xuchu Weng
}

Received: 27 March 2009 / Accepted: 29 July 2009 / Published online: 20 August 2009

(C) Springer-Verlag 2009

\begin{abstract}
The present study investigated whether offset cues have the same attentional consequences in the spatial Stroop effect as onset cues. Experiments 1 and 2 compared the attentional effects of onset-offset cues versus offset cues on the spatial Stroop effect, whereas Experiment 3 compared the attentional effects of onset versus offset cues. Across these experiments, independent of cue type (onsetoffset or onset vs. offset) and even at long stimulus-onset asynchrony, attentional cueing did not revert into inhibition of return and was modulated by spatial Stroop with greater cueing effects for incongruent arrow's direction and position. In addition, onset-offset or onset and offset cues produced comparable cueing effects in the location-direction congruent condition, and onset-offset or onset cues produced greater facilitation than offset cues in the incongruent condition. From a different perspective, peripheral cueing modulated the spatial Stroop effect in the same direction for onset-offset or onset and offset cues, although the reduction in spatial Stroop at cued locations was smaller with offset than with onset-offset or onset cues.
\end{abstract}

C. Luo $\cdot$ X. Fu $(\varangle) \cdot$ X. Weng $(\square)$

State Key Laboratory of Brain and Cognitive Science, Institute of Psychology, Chinese Academy of Sciences, 4A Datun Road, Chaoyang District, 100101 Beijing, China e-mail: fuxl@psych.ac.cn

X. Weng

e-mail: wengxc@psych.ac.cn

C. Luo

Graduate University of Chinese Academy of Sciences, Beijing, China

J. Lupiáñez

University of Granada, Granada, Spain
Keywords Onset-offset cue · Onset cue · Offset cue · Spatial Stroop effect

\section{Introduction}

In the cluttered and dynamic visual environments, there is a continuous flow of new objects and events appearing, and old objects and events disappearing. To determine whether some action must be performed in response to these objects and events, humans must obtain information on the size, color, speed and trajectory of the object (Pratt \& Hirshhorn, 2003). Perceptual processes are responsible for creating representations of these objects and events and continuously updating these representations to reflect any relevant changes in the surrounding environment. Attention processes modulate the accessibility of information within the perceptual representation and control its sampling (Samuel $\&$ Weiner, 2001). Therefore, it is important for attention to be reflexively oriented to abruptly appearing new objects or old disappearing objects.

There has been extensive work on how attention shifts as a function of the appearance of a new object or event (onset). Posner and Cohen (1984) examined the effect of peripheral onset cues on the time to detect a subsequent peripheral target. They observed that targets were detected faster at locations where an onset cue was presented (i.e., cued locations) than at uncued locations, when the cue-target stimulus-onset asynchrony (SOA) was less than $300 \mathrm{~ms}$, whereas with cue-target SOAs longer than $300 \mathrm{~ms}$, detection responses were slower for targets at the cued locations. The early facilitatory effect of the onset cue is known as the attentional cueing effect and has been hypothesized to result from the onset cue automatically capturing attention. The later inhibitory effect is known as inhibition 
of return (IOR; Posner, Rafal, Choate, \& Vaughan, 1985). The biphasic pattern of early facilitation followed by late inhibition at the cued location has been interpreted by some researchers as the mark of attentional orienting (e.g., Danziger, Kingstone, \& Ward, 2001; Funes, Lupiáñez, \& Milliken, 2007). Nevertheless, depending on the demands of the tasks, the IOR effect can only appear at longer SOAs (Lupiáñez, Milan, Tornay, Madrid \& Tudela, 1997) or may not appear at all (Lupiáñez \& Funes, 2005).

However, there has been less literature examining the effect of offset cues on the allocation of attention in the visual field. Some of them have investigated the biphasic pattern of early facilitation, followed by late inhibition at locations cued with offset cues. Using variations of the Posner and Cohen (1984) paradigm with offset cues (i.e., the disappearance of an object) and SOAs of 100, 200,300, 500 and 800 ms, Gawryszewski, Thomaz, Machado-Pinheiro and Sant'Anna (1994) observed the inhibitory effects at all SOAs. A similar pattern of results, but with much more complex displays, was observed by Samuel and Weiner (2001). Also, with simultaneously presented onset and offset cues, Pratt and Hirshhorn (2003) found that the onset cue generated early facilitation and later occurring IOR, whereas the offset cue generated significant inhibition at all, but the earliest, SOA. Across three experiments, Riggio, Bello and Umilta (1998) found varying early facilitation effects, but strong late inhibitory effects for both onset and offset cues.

Other studies have investigated whether object offset has the same attentional consequence as object onset. Using variations of the Posner cueing paradigm, Pratt and McAuliffe (2001) found that onset and offset cues produced equivalent facilitation effects at short SOAs and IOR at long SOAs. Also, Birmingham and Pratt (2005) did not find differences in IOR across several locations sequentially cued by either onsets or offsets. In line with these findings, Pratt, Theeuwes and Donk (2007) showed that various aspects of the preview effect that had previously been found with onset stimuli also occurred with offset stimuli. However, Boot, Kramer and Peterson (2005) found that incorrect pro-saccades are more likely to be triggered by onset distractors than offset distractors, as are incorrect anti-saccades (Pratt \& Trottier, 2005). In addition, Brockmole and Henderson (2005) have also shown that onsets that occur in real life scenes have more robust attention-capturing properties than offsets.

Vingilis-Jaremko, Ferber and Pratt (2008) examined visual prior entry (determining which of two stimuli appeared first) and prior exit (determining which of two stimuli disappeared first) effects with a temporal order judgment (TOJ) task. Besides onset and offset targets, the preceding cues were also either onset or offset stimuli. These authors observed typical, and equivalent, prior entry effects regardless of whether onset or offset cues preceded the onset targets. Moreover, they observed larger prior exit effects for offset targets, which were interpreted as offset cues leading to greater capture effects than onset cues. Thus, although some studies have shown similar cueing effects for onset and offset cues, others have found different effects for the two cue types. The task to be performed with the target, together with the attentional control setting generated in accordance to it, have been argued as the reason for the different cueing effects for the two type of cues.

In the present study, we employed a different research strategy to investigate the nature of the attentional capture produced by onset and offset cues, i.e., how they modulate spatial processing. Thus, the focus of the present study was to determine whether object offset has the same attentional consequences as object onset in the spatial Stroop task. As an example of the spatial Stroop task, a left-pointing or right-pointing arrow target is presented randomly to the left or right side of a fixation point. Although participants are required to discriminate the direction of the arrow while ignoring its location, they typically make faster and more accurate responses to congruent stimuli (i.e., a right-pointing arrow on the right) than to incongruent ones (i.e., a leftpointing arrow on the right) (Funes et al., 2007; Lupiáñez \& Funes, 2005; Taylor \& Ivanoff, 2005). The spatial Stroop effect seems to reflect a conflict between two streams of stimulus information, when the irrelevant stimulus dimension (location information) is incongruent with its relevant dimension (the direction of the arrow) (Lu \& Proctor, 1995).

In recent years, almost all studies investigating the relation between the spatial Stroop and attention have used object onset (specifically, object onset-offset) to examine the role of attention capture on the spatial code that produces the spatial Stroop effect (e.g., Danziger et al., 2001; Funes \& Lupiáñez, 2003; Lupiáñez \& Funes, 2005; Funes et al., 2007). The typical finding has been that peripheral attentional orienting does modulate the spatial Stroop effect so that the effect is reduced at the attended location, this modulation varying with cue-target SOA (Lupiáñez \& Funes, 2005; Funes et al., 2007). Three possible explanations have emerged to explain attentional modulation in this task (Danziger et al., 2001; Lupiáñez \& Funes, 2005).

According to the attention shift account (Rubichi, Nicoletti, Iani, \& Umilta, 1997; Stoffer, 1991), attention shifts generate spatial codes relative to the prior position of attention. This spatial code decays rapidly. Therefore, if attention has been moved toward the cued location before the appearance of the target, the spatial code generated by the cue will have dissipated, and no additive movement of attention toward the target location will be necessary, so that no location code will be activated when the target is presented. Therefore, no spatial Stroop effect would be 
measured. This account can predict the modulation of cueing over the spatial Stroop effect, but it cannot explain why a significant spatial Stroop effect (although reduced) is still observed at the attended location and why this modulation varies with cue-target SOA (Danziger et al., 2001; Lupiáñez \& Funes, 2005; Funes et al., 2007). In any case, the account would predict that the reduction in spatial Stroop would be greater at longer SOAs (when the spatial code generated by the cue has completely disappeared), whereas the opposite tendency has been reported (i.e., greater reduction at shorter SOAs, with smaller or no reduction at longer SOAs) (Funes, Lupiáñez \& Milliken, 2008; Lupiáñez and Funes, 2005).

Recently, Danziger et al. (2001) proposed a new version of the referential coding account to explain the modulation of cueing over the spatial congruency effect. This account states that the target may be coded relative to two simultaneous objects of reference, the central fixation object and the lateralized cue object. On invalidly cued trials (i.e., when the cue appears at the location opposite to the target), the target would be coded relative to both the lateralized cue object and the central fixation object, while on the validly cued trials the target would be coded only relative to the central fixation object, because it would be coded as "same" relative to the cue. This would explain why the spatial congruency effects are smaller for validly cued targets than for oppositely cued ones.

However, with this account, it would be difficult to explain some findings observed with similar tasks. For example, Funes and Lupiáñez (2003) used a similar Simon-Stroop task, but added a neutral condition in which no cue was presented. They found that spatial cues modulated the spatial congruency effect, as the magnitude of spatial congruency was significantly smaller on cued trials $(27 \mathrm{~ms})$ than on neutral trials $(44 \mathrm{~ms})$. Both were smaller than at oppositely cued ones (58 ms). This outcome was not compatible with the account proposed by Danziger et al. (2001) that predicted similar spatial Stroop effects for cued and no-cue conditions, because in both cases the targets were equally coded as "left-right" only relative to the central fixation object.

Similarly, Lupiáñez and Funes (2005) found that spatial cues reduced the spatial Stroop (stimulus-stimulus, S-S) effects that were measured when the arrow target appeared in the vertical axis, but did not influence the Simon (stimulus-response, S-R) effects that were measured when the arrow appeared in the horizontal axis. The absence of this reduction on Simon tasks (see also Hommel, 1993a; Ivanoff, Klein, \& Lupiáñez, 2002; Proctor, Lu, \& Van Zandt, 1992; Verfaellie, Bowers, \& Heilman, 1988; Zimba $\&$ Brito, 1995), are not directly predicted by the referential coding account (Danziger et al., 2001). On the contrary, the account predicts the same reduction on cued trials for spa- tial Stroop and Simon effects. Consequently, this theoretical account could not explain why the Simon effect is not modulated by spatial cuing as the spatial Stroop effect is.

Given the difficulties of the attention shift and referential coding accounts in explaining these findings, Lupiáñez, Funes et al. (Funes, Lupiáñez, \& Milliken, 2007; Lupiáñez $\&$ Funes, 2005) proposed an alternative explanation known as the event integration account. This framework is based on the notion of object files proposed by Kahneman, Treisman and Gibbs (1992). According to this account, an abrupt onset (peripheral cue), especially at short SOAs, can be regarded as a new perceptual object or event (Jonides \& Yantis, 1988; Yantis \& Jonides, 1996), sharing the spatial location with the target and contiguous in time with it. Assuming that spatial and temporal contiguity play an important role in event or object integration processes (Hommel, 1998; Kahneman, Treisman, \& Gibbs, 1992), the facilitation effect often observed at short cue-target SOAs could be attributed, at least in part, to rapid integration of the spatial codes for the cue and the target when they occur close in time and space (see Funes \& Lupiáñez, 2003; Funes et al., 2005; Funes et al., 2007, 2008; Lupiáñez \& Funes, 2005; Lupiáñez \& Milliken, 1999; Lupiáñez, Milliken, Solano, Weaver, \& Tipper, 2001, for discussions of event integration processes in exogenous cuing contexts). These authors assume that with the integration of cue and target spatial codes within the same event or object file, no extra spatial code is created when the target appears. This integration process thus helps to separate in time the processing of the two conflicting dimensions of the target stimulus, the spatial location and its direction, with the distracting location dimension of the arrow target being linked with an event that occurred at an earlier point in time (the cue). The separation in time of these two perceptual codes could then underlie the reduction in the spatial congruency effect observed for valid trials, as the irrelevant location dimension would have largely decayed by the time the relevant direction dimension was coded (see Hommel, 1993b, for discussion of this temporal overlap hypothesis as it applies to Simon interference). Note that cue-target event integration would not occur when the cue and target appear at different locations, as would be the case for uncued trials following peripheral non-informative cues and for no-cue trials (Funes \& Lupiáñez, 2003).

Contrary to the above reviewed studies investigating the modulation of exogenous attention as captured by peripheral onset-offset cues, few studies have investigated whether offset cues modulate the spatial Stroop effect. In fact, we are not aware of any research investigating this issue with offset cues, in spite of this manipulation being useful to differentiate between alternative theoretical accounts. Thus, the present study was aimed at investigating whether offset cues modulate the spatial Stroop effect, and whether they do 
so in a similar way to onset cues. This is important because the three above-outlined frameworks for explaining the modulation seem to make opposite predictions for offset cues, whereas they similarly predict a decrease of spatial Stroop at the location cued by a new object.

If offset cues are able to capture attention (see above), the attention shift account would predict a similar modulation for onset and offset cues, so that the spatial Stroop effect would not occur on validly cued trials for both cue types. If offset cues are able to capture attention, and thereby able to trigger the opening of an event representation, the event integration account also would predict similar modulation for onset and offset cues. However, the referential coding account would predict that offset cues will also modulate spatial Stroop, but in the direction opposite to that observed for onset cues. This is because for offset cues, the target would be coded only relative to the central cross on invalidly cued trials, since the lateralized box at the opposite side disappears as an offset cue; whereas on validly cued trials, the target would be coded relative to the central cross and the remaining lateralized box (see Figs. 1, 3, 5).

Given that peripheral cues seem to modulate spatial Stroop, but not Simon (Lupiáñez \& Funes, 2005), up/downpointing arrows, appearing above/below fixation, were used as targets to be responded with a left/right key press, instead of the more usual left/right-pointing arrows, in order to have a pure measure of stimulus-stimulus or Stroop interference.

A second issue investigated in the present study is with regard to the question of whether onset and offset cues capture attention in the same way and with the same effectiveness, and whether the effect of cueing will be manifested to a greater or lesser extent for congruent versus incongruent targets. To the extent that onset cues might be more effective than offset cues in triggering cue-target integration processes, and considering that those object or event integration processes are responsible for cueing effects (Lupiáñez et al., 2001) and for the modulation of cueing over spatial Stroop (Funes et al., 2007), we expect greater cueing effects for onset than for offset cues, especially for incongruent targets in which perceptual integration might

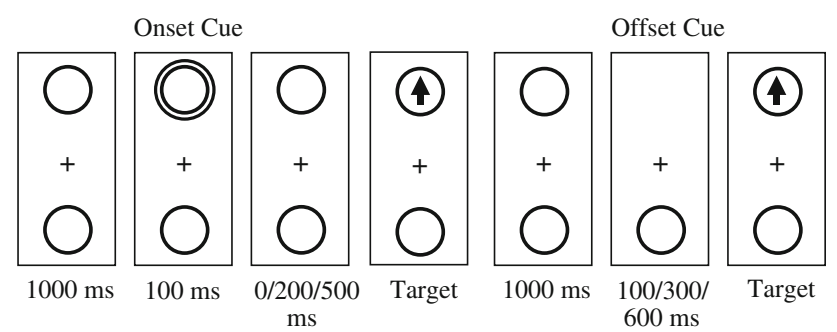

Fig. 1 The basic trial sequence used in Experiment 1. The left four columns represent onset cue condition and the right three columns represent offset cue condition be more helpful. Similarly, it could be predicted that offset cues would reduce spatial Stroop to a lesser extent than onset cues.

\section{Experiment 1}

To test the attention shift, event integration and referential coding accounts described above, a critical manipulation was made where two different cueing conditions were directly compared. For one condition (half of the trials) we presented an onset-offset cue preceding the target with a cue duration of $100 \mathrm{~ms}$, which was comparable to previous studies. For the other half of the trials, an offset cue was presented instead (offset cue condition).

\section{Method}

\section{Participants}

A total of 18 undergraduate students (10 males and 8 females) took part in the experiment for payment. All participants had normal or corrected-to-normal vision and were naive to the purpose of the experiment.

\section{Apparatus and stimuli}

Stimuli were presented on a super VGA high-resolution color monitor with a black background. A Lenovo-compatible computer, running E-PRIME 1.1 software, controlled the presentation of stimuli, timing operations and data collection. Participants viewed the monitor from a distance of approximately $57 \mathrm{~cm}$ in a dimly lit room.

The stimulus display (see Fig. 1) consisted of a fixation cross $\left(0.8^{\circ} \times 0.8^{\circ}\right.$ of visual angle $)$ at the geometrical center of the screen and two empty circular boxes (with a diameter of $3.2^{\circ}$ and a black stroke of $0.1^{\circ}$ ), centered $3.8^{\circ}$ above and below the fixation cross. They were positioned along the vertical axis. The boxes indicated the two possible positions where a black imperative arrow $\left(1.7^{\circ} \times 1.5^{\circ}\right.$ of visual angle $)$ could appear. For the onset-offset cue trials, the cue was an empty circular box (with a diameter of $3.6^{\circ}$ and a stroke of $0.1^{\circ}$ ) around one of the two black boxes, while for the offset cue trials the cue was the disappearance of one of the black boxes. The background of the display was white and all stimuli were black except that the onset-offset cue was red.

\section{Procedure and design}

For all trials, the display sequence in a trial differed depending on whether an onset-offset or an offset cue was presented. Two typical trial sequences for each cue are illustrated in Fig. 1. 
The trials began with the fixation cross and the two boxes. In the case of onset-offset cue trials, after 1,000 ms, the cue flickered around one of the two boxes with equal probability for $100 \mathrm{~ms}$. Following a further interval of 0 , 200 , or $500 \mathrm{~ms}$ from cue onset (depending on the SOA), the imperative arrow appeared in one of the two boxes. However, in the case of offset cue trials, after 1,000 ms, one of the two boxes disappeared with equal probability. Following a further interval (depending on the SOA) of 100, 300 or $600 \mathrm{~ms}$ from the disappearance of the box, the imperative arrow appeared at the location where the box disappeared or in the remaining box. The arrow remained visible until the participant responded or for $1,500 \mathrm{~ms}$ if no response was emitted. Then the next trial began. The interval between trials was $1,000 \mathrm{~ms}$ and the screen remained white throughout this interval (see Fig. 1).

There were two sessions of 432 trials each, with a rest interval of $5 \mathrm{~min}$ between them. Each session consisted of two large blocks, which corresponded respectively with onset-offset cue and offset cue, having a rest interval of $30 \mathrm{~s}$ between them and the order being randomized. Each large block included one practice block of 24 trials followed by three test blocks of 64 trials. Each test block corresponded with one SOA and their order was randomized. All participants were instructed to complete the two sessions of trials. Responses were made with the index fingers of both hands, pressing the $\mathrm{C}$ and $\mathrm{M}$ keys on the computer keyboard for left and right responses, respectively.

In one session, the task was to press the $\mathrm{C}$ key when the arrow pointed up, and to press the M key when it pointed down, regardless of the arrow's location, while the reverse mapping was used in the other session, the order being counterbalanced across participants. The response keys and computer screen were aligned such that the fixation point and the midway point between the two response keys were on the participant's sagittal midline. Participants were firmly instructed to maintain fixation and to respond to the targets as quickly and accurately as possible.

The experiment had a 2 (cue type: onset-offset, offset) $\times 2$ (cueing: valid, invalid) $\times 2$ (spatial Stroop: congruent, incongruent $) \times 3$ (SOA: $100,300,600 \mathrm{~ms})$ design, with 32 observations per experimental condition.
In all conditions, trials in which the arrow target appeared in the same location as the cue events will be referred to as valid; trials in which the arrow target appeared in the opposite location will be referred to as invalid.

\section{Results}

Mean correct RTs and percentages of errors (PE) were calculated for each participant as a function of cue type (2, onset-off, offset), cueing (2, valid, invalid), spatial Stroop (2, congruent, incongruent) and SOA (3, 100, 300, $600 \mathrm{~ms})$ (see Table 1). Mean RTs and PE data were submitted to separate analyses of variance (ANOVAs).

The ANOVA on RTs revealed a main effect of cueing, $F(1,17)=125.147, p<.001$, and a main effect of spatial Stroop, $F(1,17)=69.857, p<.001$. Cueing interacted with spatial Stroop, $F(1,17)=23.737, p<.001$, with smaller spatial Stroop effects on the validly cued trials $(15 \mathrm{~ms})$ than on the invalidly cued trials $(32 \mathrm{~ms})$. Cue type interacted with SOA, $F(2,34)=4.525, p=.018$, but not with cueing, $F(1,17)=2.616, p=0.124$. Moreover, there was a significant three-way interaction between cue type, cueing and spatial Stroop, $F(1,17)=5.720, p=.029$. No other effects were significant.

To disentangle this three-way interaction, and in line with the first purpose of the present study, a separate ANOVA for each cue type was performed, with cueing, spatial Stroop and SOA as within-participants variables (Fig. 2).

The onset-offset cue analysis revealed main effects of two variables, cueing and spatial Stroop, $F(1,17)=75.220$, $p<.001, F(1,17)=102.706, p<.001$. As in previous studies (Lupiáñez \& Funes, 2005; Funes et al., 2007), the interaction between cueing and spatial Stroop was significant, $F(1,17)=21.730, p<.001$, with smaller spatial Stroop effect on valid trials $(13 \mathrm{~ms})$ than on invalid trials $(37 \mathrm{~ms})$, although both effects were significant, $F(1,17)=15.65$, $p=.001 ; F(1,17)=90.84, p<.001$.

The offset cue analysis revealed main effects of three variables, cueing, spatial Stroop, $F(1,17)=56.201$, $p<.001, F(1,17)=25.490, p<.001$, and SOA, $F(2,34)=$ $4.807, p=.015$. More importantly, the interaction of most

Table 1 Mean reaction time (in ms) and mean percentage of error as a function of cue type, cueing, spatial Stroop and SOA for Experiment 1

\begin{tabular}{|c|c|c|c|c|c|c|c|c|}
\hline & \multicolumn{4}{|l|}{ Onset-offset } & \multicolumn{4}{|l|}{ Offset } \\
\hline & \multicolumn{2}{|l|}{ Congruent } & \multicolumn{2}{|l|}{ Incongruent } & \multicolumn{2}{|l|}{ Congruent } & \multicolumn{2}{|l|}{ Incongruent } \\
\hline & Valid & Invalid & Valid & Invalid & Valid & Invalid & Valid & Invalid \\
\hline $100 \mathrm{~ms} \mathrm{SOA}$ & $501(3.9 \%)$ & $519(2.3 \%)$ & $521(2.8 \%)$ & $551(4.7 \%)$ & $495(2.5 \%)$ & $512(1.5 \%)$ & $512(2.8 \%)$ & $533(4.3 \%)$ \\
\hline $300 \mathrm{~ms} \mathrm{SOA}$ & $488(2.0 \%)$ & $507(2.5 \%)$ & $493(3.7 \%)$ & $546(4.1 \%)$ & $495(3.3 \%)$ & $517(2.2 \%)$ & $508(2.8 \%)$ & $546(4.9 \%)$ \\
\hline $600 \mathrm{~ms} \mathrm{SOA}$ & $488(2.2 \%)$ & $509(3.0 \%)$ & $502(3.5 \%)$ & $551(5.4 \%)$ & $507(1.8 \%)$ & $527(3.2 \%)$ & $529(3.7 \%)$ & $556(3.2 \%)$ \\
\hline
\end{tabular}


Fig. 2 Mean reaction time (in $\mathrm{ms}$ ) as a function of cue type, cueing, spatial Stroop and SOA in Experiment 1

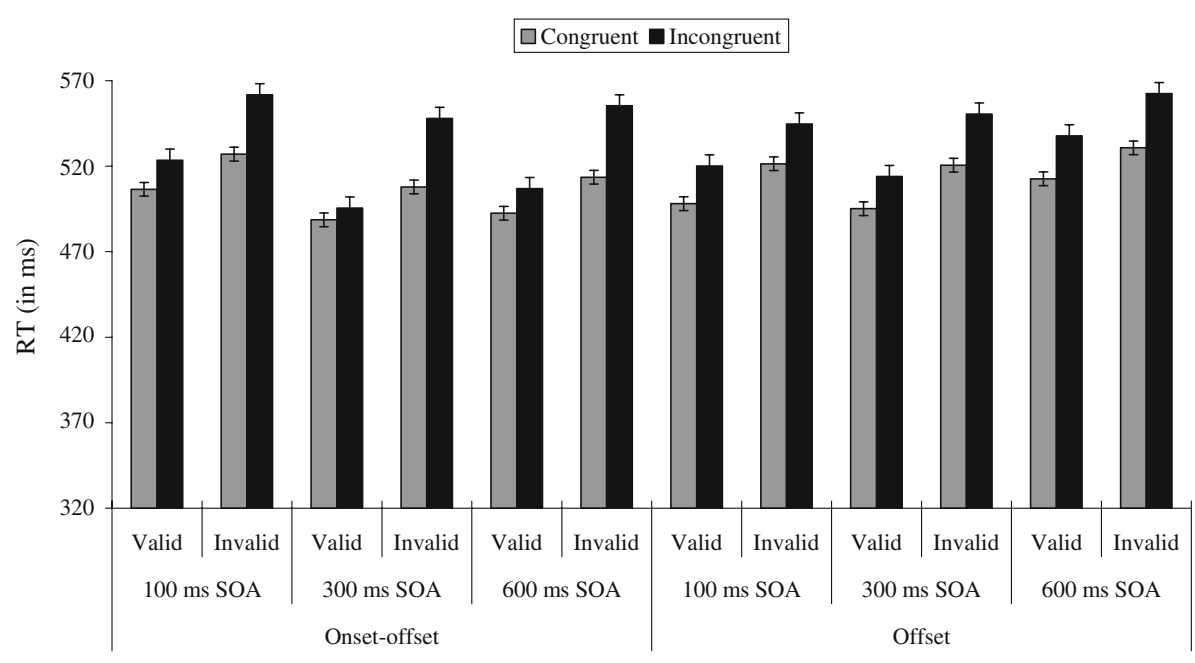

concern between cueing and spatial Stroop was significant, $F(1,17)=4.500, p=.049$, with smaller spatial Stroop effect on valid trials $(17 \mathrm{~ms})$ than on invalid trials $(26 \mathrm{~ms})$, both being significant, $F(1,17)=11.73, p=.003 ; \quad F(1,17)=$ $34.03, p<.001$.

In addition, the comparison of the magnitude of the reduction in the spatial Stroop effect by cueing with onsetoffset cues to that observed with offset cues showed that the latter $(9 \mathrm{~ms})$ was smaller than the former $(24 \mathrm{~ms})$, $t(17)=2.392, p=.029$.

In line with the second purpose of the present study and to separate the three-way interaction in a different way, a separate ANOVA was performed for congruent condition and incongruent conditions, with cue type, cueing and SOA as within-participants variables.

The analysis of the congruent condition data revealed a significant main effect of cueing, $F(1,17)=35.292$, $p<.001$, and a marginal significant interaction between cue type and SOA, $F(2,34)=3.148, p=.056$. Importantly, the interaction between cue type and cueing was not significant $(F<1)$, and no other effects were significant.

The analysis of the incongruent condition data revealed a significant main effect of cueing, $F(1,17)=174.208$, $p<.001$. Cue type interacted with SOA, $F(2,34)=5.028$, $p=.012$. In sharp contrast to the congruent condition, cue type interacted with cueing, $F(1,17)=7.918, p=.012$, with smaller cueing effect with offset cues $(29 \mathrm{~ms})$ than with onset cues (44 ms).

In addition, the comparison of the cueing effect between the congruent and incongruent conditions revealed that the former $(20 \mathrm{~ms})$ was smaller than the latter $(37 \mathrm{~ms})$, $t(17)=4.872, p<.001$.

In the error analysis, there was a main effect of spatial Stroop, $F(1,17)=10.325, p=.005$, with lower error rate on congruent trials $(2.5 \%)$ than on incongruent trials $(3.8 \%)$. There was an interaction between cueing and spatial Stroop, $F(1,17)=5.386, p=.033$, with smaller spatial
Stroop effect on validly cued trials (.6\%) than on invalidly cued trials $(2.0 \%)$. The latter was significant, $F(1,17)=$ $10.12, p=.005$, whereas the former was not $F(1,17)=3.11$, $p=.094$.

\section{Discussion}

As in Lupiáñez and Funes (2005) and Funes et al. (2007), the cueing effects with onset-offset cues did not revert to IOR even at the $600 \mathrm{~ms}$ SOA. Also, as with onset-offset cues, the cueing effects with offset cues did not revert to IOR, which is inconsistent with some previous studies that showed early inhibition with offset cues (i.e., with a $100 \mathrm{~ms}$ SOA) (e.g., Gawryszewski et al., 1994; Riggio et al., 1998; Pratt \& Hirshhorn, 2003; Samuel \& Weiner, 2001). Moreover, attentional cueing effects were modulated by spatial Stroop with smaller effects for congruent than for incongruent targets, this modulation taking place with both onset-offset and offset cues.

Importantly, as observed in previous studies (e.g., Riggio et al., 1998; Pratt \& McAuliffe, 2001; VingilisJaremko et al., 2008), there was no advantage for onsetoffset cues over offset cues when the direction of the target arrow and its position were congruent. However, when they were incongruent, greater facilitation effects were observed with onset-offset cues than with offset cues, which was consistent with some previous studies (e.g., Boot et al., 2005; Brockmole \& Henderson, 2005).

The spatial Stroop effect was modulated by cueing with onset-offset cues, replicating previous studies (Lupiáñez $\&$ Funes, 2005; Funes et al., 2007, 2008). More importantly, the spatial Stroop effect was also modulated by cueing with offset cues. Although the magnitude of the modulation was smaller with offset than with onset-offset cues, reduced spatial Stroop was also observed at locations validly cued by offset cues, as compared to invalid offset cued locations. 


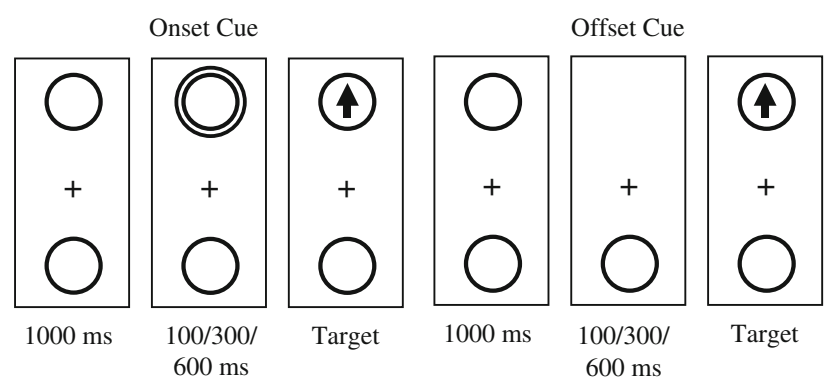

Fig. 3 The basic trial sequence used in Experiment 2. The left three columns represent onset cue condition and the right three columns represent offset cue condition

\section{Experiment 2}

In Experiment 1, we tried to equate the physical characteristics of onset-offset and offset cues, including the dynamic change. There was an important difference between the two types of cues regarding the duration of the cue. For onsetoffset cues, the cue duration was defined as $100 \mathrm{~ms}$, whereas for the offset cue, the cue duration was equal to the SOA. The aim of this manipulation was to make it comparable to previous studies (e.g., Danziger et al., 2001; Lupiáñez \& Funes, 2005; Funes et al., 2007, 2008). However, given that cue duration was different for the two types of cues, it is not clear whether the different cueing effects observed for onset-offset versus offset cues were due to the nature of the cue or rather to cue duration. Thus, in this experiment we attempted to rule out any possible role of cue duration, by making onset-offset cues also coincide with SOA (see Fig. 3).

\section{Method}

\section{Participants}

Seventeen undergraduate students (4 males and 13 females) and two postgraduate students (1 male and 1 female) took part in the experiment for payment. All participants had normal or corrected-to-normal vision and were naive to the purpose of the experiment.

\section{Apparatus, stimuli, procedure and design}

The apparatus, stimuli, procedure and design were identical to Experiment 1, except that for onset-offset cue we changed the cue duration to 300 and $600 \mathrm{~ms}$ at the 300 and $600 \mathrm{~ms}$ SOAs, respectively.

Results and discussion

Mean correct RTs and percentages of errors per experimental condition are presented in Table 2. Mean RT and errors were submitted to separate analyses of variance (ANOVAs) with four within-participants variables: cue type (2, onset-offset, offset), cueing (2, valid, invalid), spatial Stroop (2, congruent, incongruent) and SOA (3, 100, $300,600 \mathrm{~ms}$ ).

The ANOVA performed on RTs revealed main effects of cueing, $F(1,18)=60.395, p<.001$, and spatial Stroop, $F(1,18)=53.906, p<.001$. Cueing interacted with spatial Stroop, $F(1,18)=19.687, p<.001$, and SOA, $F(1,18)=$ $3.586, p=.038$. Spatial Stroop interference was smaller on validly cued trials $(12 \mathrm{~ms})$ than on invalidly cued trials $(33 \mathrm{~ms})$. Cue type interacted with spatial Stroop, $F(1,18)=$ 4.924, $p=.040$, with larger spatial Stroop interference on onset-offset trials $(26 \mathrm{~ms})$ than that on offset trials $(19 \mathrm{~ms})$, $t(18)=2.219, p=.040$. As in Experiment 1, the interaction between cue type, cueing and spatial Stroop was significant, $F(1,18)=7.486, p=.014$. No other effects were significant (Fig. 4).

To disentangle this interaction, and in terms of the first purpose of the present study, a separate ANOVA was performed for each cue type, with cueing, spatial Stroop and SOA as within-participants variables.

The onset-offset cue analysis revealed two main effects of two variables, cueing and spatial Stroop, $F(1,18)=$ $43.992, p<.001, F(1,18)=55.450, p<.001$. The interaction of most concern between cueing and spatial Stroop was significant, $F(1,18)=30.248, p<.001$, with smaller spatial Stroop effect on valid trials $(12 \mathrm{~ms})$ than on invalid trials $(41 \mathrm{~ms})$, although both effects were significant, $F(1,18)=$ $8.65, p=.009 ; F(1,18)=75.97, p<.001$, respectively.

The offset cue analysis also revealed three main effects, cueing and spatial Stroop, $F(1,18)=22.111, p<.001$,

Table 2 Mean reaction time (in ms) and mean percentage of error as a function of cue type, cueing, spatial Stroop and SOA for Experiment 2

\begin{tabular}{|c|c|c|c|c|c|c|c|c|}
\hline & \multicolumn{4}{|l|}{ Onset-offset } & \multicolumn{4}{|l|}{ Offset } \\
\hline & \multicolumn{2}{|l|}{ Congruent } & \multicolumn{2}{|l|}{ Incongruent } & \multicolumn{2}{|l|}{ Congruent } & \multicolumn{2}{|l|}{ Incongruent } \\
\hline & Valid & Invalid & Valid & Invalid & Valid & Invalid & Valid & Invalid \\
\hline $100 \mathrm{~ms} \mathrm{SOA}$ & $484(2.5 \%)$ & $490(1.4 \%)$ & $494(3.5 \%)$ & $535(5.2 \%)$ & $483(4.2 \%)$ & $488(1.7 \%)$ & $491(3.9 \%)$ & $519(4.3 \%)$ \\
\hline $300 \mathrm{~ms} \mathrm{SOA}$ & $467(1.6 \%)$ & $485(1.1 \%)$ & $483(3.0 \%)$ & $522(5.6 \%)$ & $477(2.7 \%)$ & $499(2.1 \%)$ & $491(2.2 \%)$ & $524(3.7 \%)$ \\
\hline $600 \mathrm{~ms} \mathrm{SOA}$ & $486(2.9 \%)$ & $491(1.4 \%)$ & $497(2.4 \%)$ & $529(3.8 \%)$ & $488(1.9 \%)$ & $494(1.6 \%)$ & $504(2.8 \%)$ & $513(4.8 \%)$ \\
\hline
\end{tabular}


Fig. 4 Mean reaction time (in $\mathrm{ms}$ ) as a function of cue type, cueing, spatial Stroop and SOA in Experiment 2

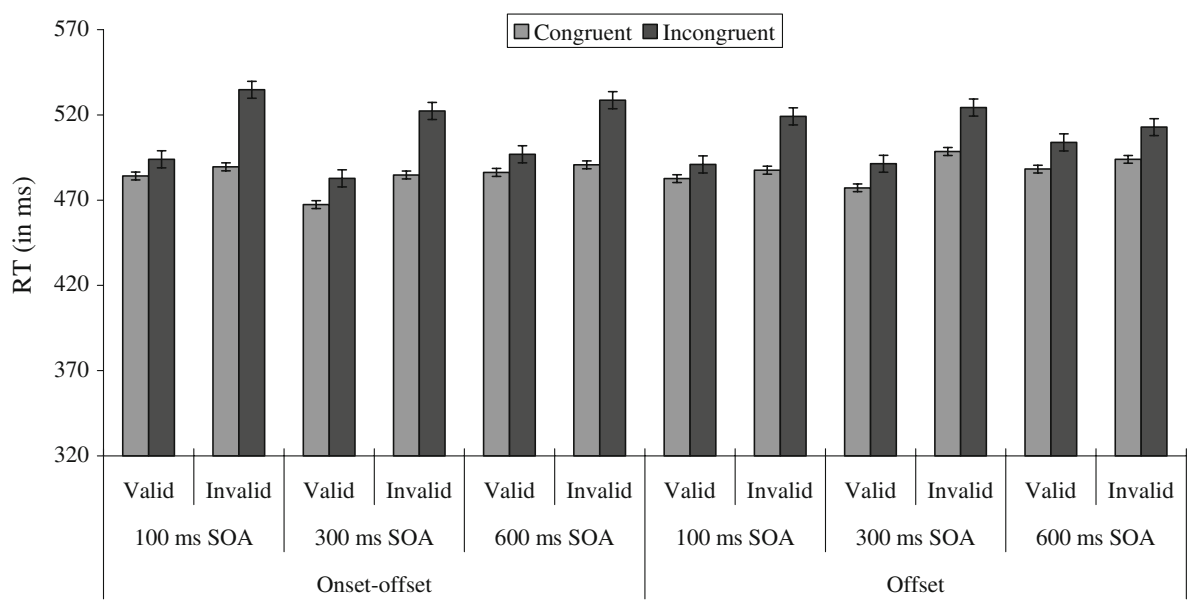

$F(1,18)=31.253, p<.001$, respectively. Cueing interacted with SOA, $F(1,18)=3.342, p=.047$, with significant cueing effect at the short SOA $(16 \mathrm{~ms}), F(1,18)=16.34$, $p=.001$, and at the medium SOA $(27 \mathrm{~ms}), F(1,18)=19.31$, $p<.001$, but not at the long SOA $(7 \mathrm{~ms}), F(1,18)=1.24$, $p=0.280$.

Importantly, the interaction of most concern between cueing and spatial Stroop was significant, $F(1,18)=4.980$, $p=.039$, with smaller spatial Stroop interference on valid trials $(12 \mathrm{~ms})$ than on invalid trials $(26 \mathrm{~ms})$, both being significant, $\quad F(1,18)=7.84, \quad p=.012 ; \quad F(1,18)=34.24$, $p<.001$.

In addition, comparing the magnitude of spatial Stroop reduction by cueing with onset cues to that with offset cues showed that the latter $(14 \mathrm{~ms})$ was smaller than the former (29 ms), $t(18)=2.392, p=.029$.

As in Experiment 1, to disentangle the three-way interaction and in line with the second aim of the present study, separate ANOVAs were performed for the congruent and the incongruent condition, with cue type, cueing and SOA as within-participants variables.

The analysis of the congruent condition data revealed a significant main effect of cueing, $F(1,18)=9.545$, $p=.006$. The interaction between cue type and cueing was not significant $(F<1)$, and no other effects were significant.

The analysis of the incongruent condition data revealed a significant main effect of cueing, $F(1,18)=66.408$, $p<.001$. However, cueing interacted now with SOA, $F(2,36)=4.366, p=.020$. Although the cueing effect was significant at the short (35 ms), medium (46 ms) and long SOA $(21 \mathrm{~ms}), F(1,18)=75.82, p<.001, F(1,18)=54.66$, $p<.001, F(1,18)=11.19, p=.004$, respectively, it was reduced at the long one. More importantly, as in Experiment 1 , and in sharp contrast with the congruent condition, cue type interacted with cueing, $F(1,18)=5.471, p=.031$, with smaller cueing effect for offset cue $(24 \mathrm{~ms})$ than for onset cue (38 ms).
In addition, the comparison of the cueing effect between the congruent and incongruent conditions revealed that the former $(10 \mathrm{~ms})$ was smaller than the latter $(31 \mathrm{~ms}), t(18)=$ 4.437, $p<.001$.

In the error rate analysis, there was a main effect of spatial Stroop, $F(1,18)=11.261, p=.004$, and an interaction between cueing and spatial Stroop, $F(1,18)=11.787$, $p=.003$, with significant spatial Stroop effect on invalidly cued trials, $F(1,18)=15.22, p=.001$, but not on validly cued trials $(F<1)$.

Overall, as in Experiment 1, the cueing effects did not invert into inhibition of return even at the $600 \mathrm{~ms}$ SOA for both cue types. For the offset cue, the cueing effect was modulated by SOA, while it did not invert into inhibition of return even at the long SOA. In fact, it remained significantly positive. Moreover, a modulation of attentional cueing effects by spatial Stroop was again observed with smaller cueing effects for congruent targets. Importantly, as in Experiment 1, similar facilitation was observed for onset and offset cues with congruent targets, whereas greater facilitation was observed for onset than for offset cues with incongruent targets. Again, the spatial Stroop effect was modulated by cueing. More importantly, the spatial Stroop effects were also modulated by cueing with offset cues. The overall pattern of results from Experiments 1 and 2 therefore shows that the change in cue duration does not affect the pattern of modulation of cueing over the spatial Stroop with onset-offset cues, and the overall effects of onsets and offsets on visual attention in the spatial Stroop task.

\section{Experiment 3}

In this experiment, we aimed to further investigate the role of onset and offset cues in the spatial Stroop task. Note that onset cues disappeared when the target appeared, thus leading to a change in the cued location at the moment the target appeared. In contrast, with offset cues, there was no 


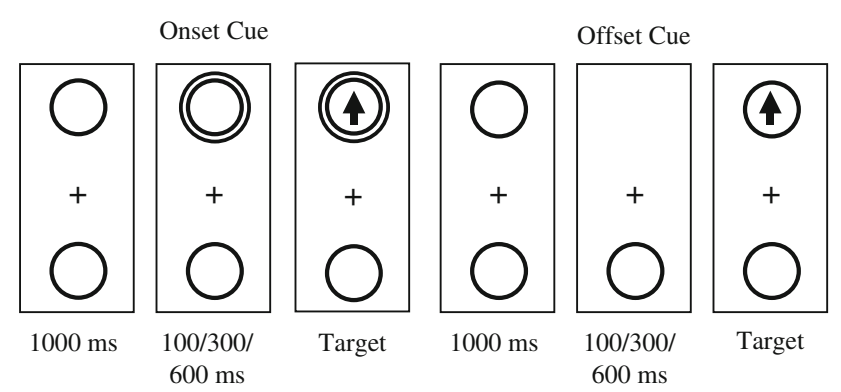

Fig. 5 The basic trial sequence used in Experiment 3. The left three columns represent onset cue condition and the right three columns represent offset cue condition

extra change in the display once the offset cue disappeared. Thus, in this new experiment, in the onset cue condition, the cue did not disappear until the trial terminated, to equate the dynamic properties of onset and offset cues.

\section{Method}

\section{Participants}

Eighteen undergraduate students (8 males and 10 females) took part in the experiment for payment. All participants had normal or corrected-to-normal vision and were naive to the purpose of the experiment.

\section{Apparatus, stimuli, procedure and design}

The apparatus, stimuli, procedure and design were identical to Experiment 2, except that in the onset cue condition the cue remained visible until the trial terminated (Fig. 5).

\section{Results and discussion}

Mean correct RTs and percentages of errors per experimental condition are presented in Table 3. RT and errors were submitted to separate analyses of variance (ANOVAs) with four within-participants variables: cue type (2, onset, offset), cueing (2, validly cued, invalidly cued), spatial Stroop (2, congruent, incongruent) and SOA (3, 100, 300, $600 \mathrm{~ms})$.
The ANOVA performed on RTs revealed main effects of cueing, $F(1,17)=38.696, p<.001$, and spatial Stroop, $F(1,17)=22.798, p<.001$. Cueing interacted with spatial Stroop, $F(1,17)=19.669, p<.001$, with smaller spatial Stroop effects on validly cued trials $(13 \mathrm{~ms})$ than on invalidly cued trials $(34 \mathrm{~ms})$. Also, cueing interacted with cue type, $F(1,17)=7.509, p=.014$, with smaller cueing effects with offset cues $(15 \mathrm{~ms})$ than with onset cues $(31 \mathrm{~ms})$. The interaction between cueing and SOA was marginally significant, $F(2,34)=3.205, p=.053$.

As in Experiment 1-2, and in line with the first purpose of the present study, a separate ANOVA was performed for each cue type, with cueing, spatial Stroop and SOA as within-participants variables.

The onset cue analysis revealed significant main effects of cueing and spatial Stroop, $F(1,17)=67.479, p<.001$, $F(1,17)=14.347, p=.001$. Cueing interacted with SOA, $F(2,34)=4.428, \quad p=.020$, although significant cueing effects were observed at each SOA; $100 \mathrm{~ms}(29 \mathrm{~ms})$, $F(1,17)=69.77, p<.001,300 \mathrm{~ms}(43 \mathrm{~ms}), F(1,17)=38.00$, $p<.001$, and $600 \mathrm{~ms}(20 \mathrm{~ms}), F(1,17)=9.95, p=.006$.

As shown in Table 3 and Fig. 6, the interaction of most concern between cueing and spatial Stroop was significant, $F(1,17)=14.733, p=.001$, with smaller spatial Stroop effect on validly cued trials $(9 \mathrm{~ms})$ than on invalidly cued trials $(36 \mathrm{~ms})$, and only the latter being significant, $F(1,17)=29.64, p<.001$.

The offset cue analysis also revealed main effects of cueing and spatial Stroop, $F(1,17)=7.052, \quad p=.017$; $F(1,17)=20.811, p<.001$. Again, as shown in Table 3 and Fig. 6, the interaction of most concern between cueing and spatial Stroop was also significant, $F(1,17)=5.502$, $p=.031$, with smaller spatial Stroop effect on validly cued trials $(15 \mathrm{~ms})$ than on invalidly cued trials $(32 \mathrm{~ms})$, both being significant, $F(1,17)=5.48, p=.032, F(1,17)=27.53$, $p<.001$, respectively.

In addition, comparing the magnitude of spatial Stroop reduction by cueing with onset versus offset cues showed that the latter $(17 \mathrm{~ms})$ was numerically smaller than the former $(27 \mathrm{~ms})$, although not significantly different, $t(17)=1.028, p=0.318$.

As in Experiment 1-2, and in line with the second purpose of the present study, a separate ANOVA was

Table 3 Mean reaction time (in ms) and mean percentage of error as a function of cue type, cueing, spatial Stroop and SOA for Experiment 3

\begin{tabular}{|c|c|c|c|c|c|c|c|c|}
\hline & \multicolumn{4}{|l|}{ Onset } & \multicolumn{4}{|l|}{ Offset } \\
\hline & \multicolumn{2}{|l|}{ Congruent } & \multicolumn{2}{|l|}{ Incongruent } & \multicolumn{2}{|l|}{ Congruent } & \multicolumn{2}{|l|}{ Incongruent } \\
\hline & Valid & Invalid & Valid & Invalid & Valid & Invalid & Valid & Invalid \\
\hline $100 \mathrm{~ms} \mathrm{SOA}$ & $515(3.9 \%)$ & $527(4.3 \%)$ & $524(4.7 \%)$ & $571(7.7 \%)$ & $508(4.1 \%)$ & $514(3.3 \%)$ & $519(6.2 \%)$ & $544(7.7 \%)$ \\
\hline $300 \mathrm{~ms} \mathrm{SOA}$ & $507(4.1 \%)$ & $537(5.7 \%)$ & $511(5.2 \%)$ & $568(8.9 \%)$ & $517(2.7 \%)$ & $523(3.5 \%)$ & $534(5.4 \%)$ & $557(7.2 \%)$ \\
\hline $600 \mathrm{~ms} \mathrm{SOA}$ & $518(4.7 \%)$ & $528(4.6 \%)$ & $532(5.4 \%)$ & $562(5.7 \%)$ & $517(2.9 \%)$ & $523(4.9 \%)$ & $534(5.8 \%)$ & $554(7.7 \%)$ \\
\hline
\end{tabular}


Fig. 6 Mean reaction time (in $\mathrm{ms}$ ) as a function of cue type, cueing, spatial Stroop and SOA in Experiment 3

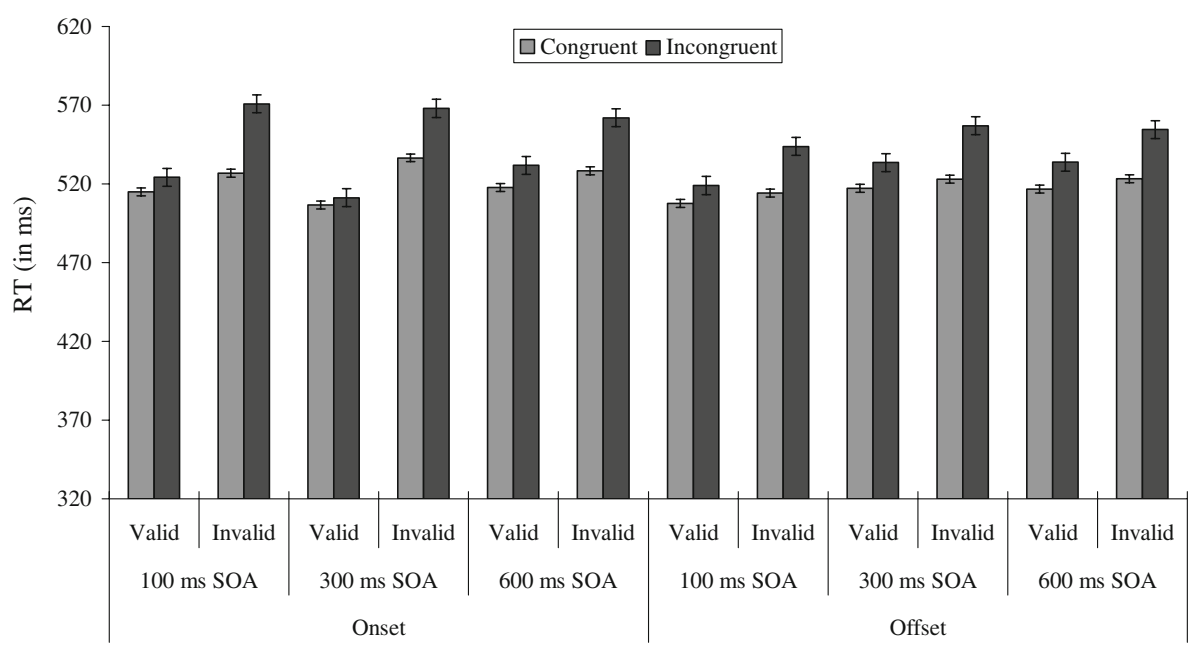

performed for the congruent and incongruent conditions of spatial Stroop with cue type, cueing and SOA as withinparticipants variables, respectively. The analysis of the congruent condition data revealed a significant main effect of cueing, $F(1,17)=9.566, p=.007$. The interaction between cue type and cueing was not significant, $F(1,17)=2.366$, $p=0.142$, and no other effects reached significance.

The analysis of the incongruent condition data revealed a significant main effect of cueing, $F(1,17)=47.044$, $p<.001$. The interaction between cueing and SOA was significant, $F(2,34)=4.410, p=.020$, with smaller cueing effect at the long SOA, although it was significant at all short $(35 \mathrm{~ms})$, medium $(40 \mathrm{~ms})$ and long SOAs $(25 \mathrm{~ms})$, $F(1,17)=43.96, \quad p<.001, \quad F(1,17)=95.10, \quad p<.001$, $F(1,17)=12.37, p=.004$, respectively. In sharp contrast to the congruent condition, cue type interacted with cueing, $F(1,17)=6.624, p=.020$, with smaller cueing effect for offset cues ( $23 \mathrm{~ms}$ ) than for onset cues $(45 \mathrm{~ms})$.

In addition, the comparison of the cueing effect between the congruent and incongruent conditions revealed that the former $(12 \mathrm{~ms})$ was smaller than the latter $(34 \mathrm{~ms})$, $t(17)=4.435, p<.001$.

In the error rate analysis, only the main effects of cueing, $F(1,17)=29.435, \quad p<.001, \quad$ and spatial Stroop, $F(1,17)=8.105, p=.011$, were significant. No other effects were significant.

Overall, as in Experiment 1 and 2, the cueing effects did not invert into inhibition of return even at the $600 \mathrm{~ms} \mathrm{SOA}$, regardless of the cue type. Also, a similar modulation of attentional cueing effects by spatial Stroop was observed with smaller cueing effects for congruent targets. Importantly, as in Experiment 1 and 2, there was no advantage of onset cues over offset cues with congruent targets, whereas greater facilitation was observed for onset than for offset cues with incongruent targets. Again, and more importantly, the spatial Stroop effect was modulated by cueing with onset and offset cues.

\section{Comparative analysis}

To compare the results of the three experiments, an ANOVA was performed on the mean correct RTs from the three experiments, with experiment (Experiments 1,2,3) as a between-participant variable and cue type (onset-offset or onset, offset), cueing (validly cued, invalidly cued), spatial Stroop (congruent, incongruent), and SOA (100, 300, $600 \mathrm{~ms}$ ) as within-participant variables. There were two significant main effects, cueing, $F(1,52)=191.599$, $p<.001$, and spatial Stroop, $F(1,52)=118.870, p=.001$, whereas neither the main effect of experiment nor the interaction of experiment with any of the other factors was significant (all $p \mathrm{~s} \geq 0.164$ ). Cueing interacted with cue type, $F(1,52)=11.190, \quad p=.002, \quad$ spatial Stroop, $F(1,52)=$ $60.467, p<.001$, and SOA, $F(2,104)=6.360, p=.002$. Importantly, the three-way interaction between cue type, cueing and spatial Stroop was significant, $F(1,52)=9.923$, $p=.003$, independent of the experiment $(F<1)$.

The separate ANOVAs performed for each cue type, with experiment as a between-participant variable and cueing, spatial Stroop and SOA as within-participant variables, showed similar results to those observed for each individual experiment. The onset-offset or onset cue analysis revealed significant main effects of cueing, spatial Stroop and SOA, $F(1,52)=185.530, p<.001 ; F(1,52)=101.733, p<.001$; $F(2,104)=3.921, p=.023$, and again no main effect of experiment or interaction of experiment with any of the other factors (all $p s \geq 0.191$ ). Importantly, the interaction between cueing and spatial Stroop was significant, $F(1,52)=61.939, p<.001$, with smaller spatial Stroop on validly cued trials $(11 \mathrm{~ms})$ than on invalidly cued trials (38 ms), independent of experiment $(F<1)$.

The offset cue analysis also revealed significant main effects of cueing, spatial Stroop and SOA, $F(1,52)=58.153$, $p<.001 ; F(1,52)=74.028, p<.001 ; F(2,104)=4.688$, $p=.011$, and no main effect of experiment or interaction of 
experiment with any of the other factors (all $p s \geq 0.276$ ). Again, the interaction of most concern between cueing and spatial Stroop was also significant, $F(1,52)=14.568$, $p<.001$, with smaller spatial Stroop effect on validly cued trials $(15 \mathrm{~ms})$ than on invalidly cued trials $(28 \mathrm{~ms})$, independent of experiment $(F<1)$. Thus, as in each individual experiment, spatial Stroop was reduced by cueing the target location with either an onset or an offset. However, cueing with an onset and onset-offset led to a greater reduction $(27 \mathrm{~ms})$ than cueing with an offset $(13 \mathrm{~ms}), t(54)=2.765$, $p=.008$.

All the effects that were significant in the analyses for each individual experiment were significant in the individual omnibus ANOVA and independent of the experiment.

\section{General discussion}

The present study investigated the relation between attentional capture by onset versus offset cues and the spatial Stroop effect. In particular, we sought to determine whether the modulation of peripheral cueing over the spatial Stroop effect, which has been observed when the appearance of an onset-offset is used as cue, also occurs when an object disappearance (offset) is used as cue. Experiments 1 and 2 compared the attentional effects of onset-offset cues and offset cues in the spatial Stroop task, and Experiment 3 compared the attentional effects of onset cues and offset cues in the spatial Stroop task.

Across the three experiments, the cueing effects did not invert into inhibition of return even at the $600 \mathrm{~ms}$ SOA, regardless of the cue type. This outcome is inconsistent with previous studies (e.g., Gawryszewski et al., 1994; Samuel \& Weiner, 2001), which observed IOR with offset cues even when the SOA was shorter than $100 \mathrm{~ms}$. The absence of IOR in our experiments even at the $600 \mathrm{~ms}$ long SOA might be due to the additional perceptual conflict present in the discrimination task. The spatial congruency manipulation may need an additional perceptual effort to resolve the conflict between the relevant and irrelevant dimensions, which may increase facilitation at the validly cued location (Klein, 2000) or maintain the perceptual integration set (see Lupiáñez et al., 2001, for a discussion and a similar result using a difficult form discrimination task), both consequently delaying the appearance of IOR. Similar long-lasting facilitation effects were obtained by Lupiáñez and Funes (2005); see also Funes et al. (2007) for the spatial Stroop task and the study by Callejas, Lupiáñez, Funes and Tudela (2005) for the Flanker task.

Nevertheless, the most important finding was, in line with our hypotheses, that the spatial Stroop effect was modulated by cueing not only for onset or onset-offset cues, but also for offset cues, with smaller spatial Stroop effects on validly cued trials than on invalidly cued trials. Importantly, however, onset-offset or onset cues led to a greater reduction of spatial Stroop than offset cues. This outcome was helpful to differentiate the three accounts for the reduction of spatial Stroop by peripheral cueing.

The referential coding account proposed by Danziger et al. (2001) can easily explain the modulation of cueing over the spatial Stroop effect with onset-offset or onset cues, because on invalidly cued trials the target would be coded relative to the object cue and the central cross, while on validly cued trials the target location would be coded only relative to the central cross. However, this account cannot explain why this modulation also occurred for offset cues. According to this account, on validly cued trials, the target would be coded relative to the uncued location object (the remaining box in the screen) and the central cross, while on invalidly cued trials the target location would be coded only relative to the central cross; importantly, it will not be coded relative to the location cued by an offset, as no object will be present there when the target appears. Thus, this account should predict no modulation, or opposite modulation of spatial Stroop by offset cueing (i.e., increased interference on validly cued trials), which was obviously inconsistent with the outcome observed in the present study.

The attention shift account can easily explain the modulation of cueing over spatial Stroop with both onset-offset or onset cues and offset cues. According to this account, no spatial Stroop effect would be measured on validly cued trials, because there would be no additive movement of attention toward the target location when the target is presented, resulting in no establishment of any extra location code (or it would have disappeared by the time the target appears). However, on invalidly cued trials, attention would move from the cued location to the opposite location where the target is presented, so that a new location code would be created and a great spatial Stroop effect would be observed. However, as in the previous studies (Danziger et al., 2001; Lupiáñez \& Funes, 2005; Funes et al., 2007), with this account, it would be difficult to explain the observation in the present study of a significant (although reduced) spatial Stroop effect on validly cued trials.

Conversely, this finding thoroughly reconciles with the event integration account for the reduction of spatial Stroop effect by cueing (Funes et al., 2007; Lupiáñez \& Funes, 2005). According to this account, with valid onset-offset or onset cues, due to the spatial and temporal contiguity between the cue object and the arrow target, they could integrate together within the same object of event file (Hommel, 2004; Kahneman et al., 1992). Integration occurring on valid trials leads to the target not being coded as appearing either above or below, but as appearing within 
the object file opened by the cue, which results in the reduction of the spatial Stroop effect on validly cued trials. For invalid trials, a new object file must be created when the target appears, thus leading to simultaneous activation of location and direction codes and therefore regular spatial Stroop. Importantly, some integration processes might occur for offset cues, so that similar spatial Stroop reductions are observed, especially at short SOAs.

However, although both onset and offset cues might lead to the generation of an event code, only onset cues would generate an object file. The coincidence of both object and event representations in the case of onset cues (but not for offset cues) might lead to stronger cue-target integration processes, and therefore the magnitude of the reduction of spatial Stroop by cueing would be smaller with offset than with onset-offset or onset cues, as observed in the reported experiments.

If we look at the cueing $\times$ spatial Stroop interaction from a different perspective, the cueing effect was smaller for congruent than for incongruent targets. Also, onset and offset cues led to similar cueing effect for congruent targets, whereas onset cues produced greater cueing effects than offset cues for incongruent targets. One might be tempted to attribute this outcome to an additional perceptual effort to resolve the conflict between the relevant and irrelevant dimensions when they are incongruent. Following Klein (2000), it could be argued that when the direction of the arrow and its position are incongruent, more attentional resources may be allocated to the task, which, in turn, leads to more attention being allocated to cued locations. Essentially, as targets become more difficult to identify, the amount of attention allocated to the task increases, and this results in greater attention capture from peripheral cues. This basic notion has also been advanced as an explanation as to why IOR occurs at longer SOAs in identification than in detection tasks (Klein, 2000) and why older adults show IOR at longer SOAs than younger adults (i.e., deficits in sensory processing make target detection tasks more difficult for the elderly; Castel, Chasteen, Scialfa, \& Pratt, 2003). Evidence for this interpretation also comes from Reuter-Lorenz, Jha and Rosenquist (1996), who found more early facilitation and late IOR for blocks of trials with dim targets than for those with bright targets.

Note, however, that in our experiments the two types of targets (i.e., congruent and incongruent) were randomly mixed within the same block of trials. Therefore, the different cueing effects observed for congruent and incongruent targets cannot be explained on the basis that attention is captured differently by the cue on congruent and incongruent trials, or differently maintained on its location. Note that the cue appears before the target, and, therefore, before congruent and incongruent trials are defined. Therefore, the effect must be explained as a different manifestation of the same attentional capture.

Note also that the target was always an onset event, and, therefore, more similar to onset or onset-offset cues than to offset cues. Therefore, we might expect a greater cueing effect for the target matching the type of cue (i.e., onset cues), as a specific attentional control setting would be created for it. In fact, in a recent study (Chica, Lupiáñez, Rossi, \& Riggio, submitted), greater (or more positive) cueing effects have been observed when the cue and the target have the same type of event (either onset or offset) as compared to when they have different type. However, again this account would not explain why this greater cueing effect for onset or onset-offset cues only occurred for the incongruent targets, in spite of congruent and incongruent targets being randomly mixed within the same block of trials and, therefore, not being able to generate differential attentional control setting. Again, the effect must be rather explained as a different manifestation of the same attentional capture.

In fact, attentional capture has been shown to be more beneficial for more perceptually complex targets (Lupiáñez, Ruz, Funes, \& Milliken, 2007). According to these authors, greater benefits are obtained for targets benefiting more from spatial selection (Lupiáñez \& Chica, submitted). As stated above, onset or onset-offset cues might lead to more efficient cue-target integration processes than offset cues, due to the coincidence of both object and event representations in the former. Thus, onset or onset-offset cues would lead to greater benefits in the spatial selection of the target without the need to generate a new spatial code, which in turn would produce greater cueing effects, especially in more perceptually demanding situations such as the incongruent conditions in our experiments. Therefore, onset or onset-offset cues would produce a greater cueing effect for incongruent targets because they would be more efficient in selecting the object representation of the target that is needed to perform the analytic perceptual processing, which is in turn needed to select the target direction in the context of a competing location.

Actually, we have recently observed some evidence indicating that the modulation of cueing on spatial interference is object based, as predicted by the event integration account (Luo, Lupiáñez, Funes, \& Fu, 2009; Experiment 1). In this study, we combined a double-rectangle cueing task developed by Egly, Driver and Rafal (1994) with the spatial Stroop task used by Lupiáñez and Funes (2005) to separate the role of object-based and space-based attention on spatial codes and to further understand the role of attention in the generation and modulation of spatial codes. Participants were presented on each trial two parallel rectangles symmetrically arranged at each side of the fixation point, either vertically or horizontally, while they were instructed to identify the direction of a left-right pointing 
arrow, which could appear at one end of one of the rectangles. Shortly before the target onset, the end of one of the rectangles was flashed briefly as a cue. On $25 \%$ of the trials (valid-cue trials), the target was presented at the cued location. On the remaining trials, the target appeared at one of the two locations equally distant from the cued location (i.e., at the opposite end of the same rectangle, same object trials, or at the nearer end of the other rectangle, differentobject trials), or at the rectangle end diametrically opposite to the cued end. We observed that responses were faster on same location trials than on the other trials, indicating that location or distance from the cue affected performance (a space-based effect), and for same object targets than for different object targets, notwithstanding their equivalent distance from the same location, indicating that the rectangle object also influenced the allocation of attention: an object-based effect. Importantly, we observed that the spatial Stroop effect was modulated by object-based attention, but not by space or location-based attention. For the congruent condition, only a location-based effect was observed (i.e., responses were faster at the cued location than at uncued locations, with no difference between cued and uncued object). However, for incongruent targets, both location- and object-based effects were observed. Thus, it seems that object-based attention (perhaps through object integration processes) is especially effective in helping processing needed to solve spatial incongruence. These object integration processes seem to be especially involved when a real object is presented (i.e., with onset cues), thus explaining the greater cueing effect of onset cues with incongruent targets.

With other research paradigm, it has been also shown that cue types (onset vs. offset) can determine other attentional effects such as the disengage deficit in neglect patients (Bartolomeo, 2007). Rastelli, Funes, Lupiáñez, Duret and Bartolomeo (2008) observed that neglect patients performed as control participants with offset cues, showing attentional orienting but not disengage deficit, whereas they showed the standard disengage deficit with onset cues (excessively long RT when the cue was presented to the right and the target to the left). These results were interpreted as suggesting that the disengage deficit is object based.

One main difference between the present study and some of the previous studies investigating the relation between onset cueing and spatial Stroop (Funes et al., 2007, 2008; Lupiáñez \& Funes, 2005) is that we manipulated the different levels of SOA blocked, instead of mixed within a given block of trials. This might be responsible, at least in part, for the lack of spatial Stroop by cueing by SOA interaction, which was observed in previous studies, with the reduction of spatial Stroop on valid trials being stronger at short than at long SOAs (Funes et al., 2007, 2008). However in the present study, the reduction of spatial Stroop on valid trials seems very similar across SOAs in the three experiments. The within-block manipulation of SOA might allow participants to be easily sensitive to the relative difference between long and short time distance, thus the long SOA being perceived longer than when all trials are equally long (on block designs). Consequently, this might affect the ease of cue-target integration processes at long levels of SOA, which are more difficult to occur on mixed designs than on blocked ones. Nevertheless, more research should be carried out to test this hypothesis.

In conclusion, in the three reported experiments, we have observed that onset or onset-offset and offset cues seem to be effective in capturing attention. However, the attentional capture by onset or onset-offset cues seems to be more effective in facilitating processing than that by offset cues, especially when target discrimination processes are more important (i.e., in the incongruent condition). Similarly, onset or onset-offset cues seem to be more effective than offset cues in reducing spatial Stroop interference at the cued location. The overall pattern of results is consistent with object or event cue-target integration processes as determinant of the benefits in spatial selection driven by peripheral spatial cueing.

Acknowledgments This research was supported in part by grants from 973 Program of Chinese Ministry of Science and Technology (Grant \# 2006CB303101 and 2007CB303101) and the National Natural Science Foundation of China (Grant \# 90820305, 30500157 and 30600182). This research was also financially supported in part by the Spanish Ministry of Education and Science with research projects PSI2008-03595PSIC and CSD2008-00048.

\section{References}

Bartolomeo, P. (2007). Visual neglect. Current Opinion in Neurology, 20(4), 381-386.

Birmingham, E., \& Pratt, J. (2005). Examining inhibition of return with onset and offset cues in the multiple cueing paradigm. Acta Psychologica, 118, 101-121.

Boot, W. R., Kramer, A. F., \& Peterson, M. S. (2005). Oculomotor consequences of abrupt onsets and offsets: Onsets dominate oculomotor capture. Perception \& Psychophysics, 67, 910-928.

Brockmole, J. R., \& Henderson, J. M. (2005). Object appearance, disappearance, and attention prioritization in real-world scenes. Psychonomic Bulletin \& Review, 12, 1061-1067.

Callejas, A., Lupiáñez, J., Funes, M. J., \& Tudela, P. (2005). Modulations among the alerting, orienting and executive control networks. Experimental Brain Research, 167(1), 27-37.

Castel, A., Chasteen, A. L., Scialfa, C. T., \& Pratt, J. (2003). Adult age differences in the time course of inhibition of return. Journal of Gerontology: Psychological Sciences, 58, 256-259.

Chica, A., Lupiáñez, J., Rossi, A., \& Riggio, L. (submitted). Differential cuing effects for onset and offset cues under identical task sets.

Danziger, S., Kingstone, A., \& Ward, R. (2001). Environmentally defined frames of reference: Their sensitivity to spatial cues and attention, and their time course. Journal of Experimental Psychology: Human Perception and Performance, 27, 494-503. 
Funes, M. J., \& Lupiáñez, J. (2003). La teoría atencional de Posner: Una tarea para medir las funciones atencionales de orientación, alerta y control cognitivo y la interacción entre ellas. Psicothema, $15,260-266$

Funes, M. J., Lupiáñez, J., \& Milliken, B. (2005). The role of spatial attention and other processes on the magnitude and time course of cueing effects. Cognitive Processing-International Quarterly of Cognitive Science, 6, 98-116.

Funes, M. J., Lupiáñez, J., \& Milliken, B. (2007). Separate mechanisms recruited by exogenous and endogenous spatial cues: evidence from a spatial Stroop paradigm. Journal of Experimental Psychology: Human Perception and Performance, 33(2), 248-262.

Funes, M. J., Lupiáñez, J., \& Milliken, B. (2008). The modulation of exogenous spatial cueing on spatial Stroop interference: evidence of a set for "cue-target event segregation". Psicológica, 29, 6595.

Gawryszewski, L. G., Thomaz, T. G., Machado-Pinheiro, W., \& Sant'Anna, A. N. (1994). Onset and offset of a visual cue have different effects on manual reaction time to a visual target. Brazilian Journal of Medical and Biological Research, 27, 67-73.

Hommel, B. (1993a). The role of attention for the Simon effect. Psychological Research, 55, 208-222.

Hommel, B. (1993b). The relationship between stimulus processing and response selection in the Simon task: evidence for a temporal overlap. Psychological Research, 55, 280-290.

Hommel, B. (1998). Event files: evidence for automatic integration of stimulus-response episodes. Visual Cognition, 5(1-2), 183-216.

Hommel, B. (2004). Event files: feature binding in and across perception and action. Trends in Cognitive Sciences, 8(11), 494-500.

Ivanoff, J., Klein, R. M., \& Lupiáñez, J. (2002). Inhibition of return interacts with the Simon effect: an omnibus analysis and its implications. Perception \& Psychophysics, 64, 318-327.

Jonides, J., \& Yantis, S. (1988). Uniqueness of abrupt visual onset in capturing attention. Perception \& Psychophysics, 43, 346-354.

Kahneman, D., Treisman, A., \& Gibbs, B. (1992). The reviewing of object files: object-specific integration of information. Cognitive Psychology, 24, 175-219.

Klein, R. M. (2000). Inhibition of return. Trends in Cognitive Sciences, $4,138-147$.

Lu, C.-H., \& Proctor, R. W. (1995). The influence of irrelevant location information on performance: a review of the Simon effect and congruency effects. Psychonomic Bulletin \& Review, 2, 174-207.

Luo, C., Lupiáñez, J., Funes, M. J., \& Fu, X. (2009). Modulation of spatial Stroop by object-based attention but not by space-based attention. Quarterly Journal of Experimental Psychology.

Lupiáñez, J., \& Chica, A. B. (submitted). Inhibition of return and attentional disengagement.

Lupiáñez, J., \& Funes, M. J. (2005). Peripheral spatial cues modulate spatial Stroop interference: analyzing the "locus" of the cueing modulation. European Journal of Cognitive Psychology, 17, 727752 .

Lupiáñez, J., Milan, E. G., Tornay, F. J., Madrid, E., \& Tudela, P. (1997). Does IOR occur in discrimination tasks? Yes, it does, but later. Perception and Psychophysics, 59(8), 1241-1254.

Lupiáñez, J., \& Milliken, B. (1999). Inhibition of return and the attentional set for integrating versus differentiating information. The Journal of General Psychology, 126(4), 392-418.

Lupiáñez, J., Milliken, B., Solano, C., Weaver, B., \& Tipper, S. P. (2001). On the strategic modulation of the time course of facilita- tion and inhibition of return. The Quarterly Journal of Experimental Psychology, 54(3), 753-773.

Lupiáñez, J., Ruz, M., Funes, M. J., \& Milliken, B. (2007). The manifestation of attentional capture: facilitation or IOR depending on task demands. Psychological Research, 71(1), 77-91.

Posner, M. I., \& Cohen, Y. (1984). Components of visual orienting. In H. Bouma \& D. G. Bouwhuis (Eds.), Attention and performance $X$ : control of language processes (pp. 531-556). Hillsdale: Erlbaum.

Posner, M. I., Rafal, R. D., Choate, L. S., \& Vaughan, J. (1985). Inhibition of return: neural basis and function. Cognitive Neuropsychology, 2, 211-228.

Pratt, J., \& Hirshhorn, M. (2003). Examining the time course of facilitation and inhibition with simultaneous onset and offset cues. Psychological Research, 67, 261-265.

Pratt, J., \& McAuliffe, J. (2001). The effects of onsets and offsets on visual attention. Psychological Research, 65, 185-191.

Pratt, J., Theeuwes, J., \& Donk, M. (2007). Offsets and prioritizing the selection of new elements in search displays: more evidence for attentional capture in the preview effect. Visual Cognition, 15, $133-148$.

Pratt, J., \& Trottier, L. (2005). Pro-saccades and anti-saccades to onset and offset targets. Vision Research, 45, 765-774.

Proctor, R. W., Lu, C. H., \& Van Zandt, T. (1992). Enhancement of the Simon effect by response precuing. Acta Psychologica, 81, 53-74.

Rastelli, F., Funes, M. J., Lupiáñez, J., Duret, C., \& Bartolomeo, P. (2008). Left visual neglect: is the disengage deficit Space- or object-based? Experimental Brain Research, 187(3), 439-446.

Reuter-Lorenz, P. A., Jha, A. P., \& Rosenquist, J. N. (1996). What is inhibited in inhibition of return? Journal of Experimental Psychology: Human Perception \& Performance, 22, 367-378.

Riggio, L., Bello, A., \& Umilta, C. (1998). Inhibitory and facilitatory effects of cue onset and offset. Psychological Research, 61, 107118.

Rubichi, S., Nicoletti, R., Iani, C., \& Umilta, C. (1997). The Simon effect occurs in relation to the direction of an attention shift. Journal of Experimental Psychology: Human Perception and Performance, 5, 1353-1364.

Samuel, A. G., \& Weiner, S. K. (2001). Attentional consequences of object appearance and disappearance. Journal of Experimental Psychology: Human Perception and Performance, 27(6), 14331451.

Stoffer, T. H. (1991). Attentional focusing and spatial stimulusresponse compatibility. Psychological Research, 53, 127-135.

Taylor, T. L., \& Ivanoff, J. (2005). Inhibition of return and repetition priming effects in localization and discrimination tasks. Canadian Journal of Experimental Psychology, 59, 75-89.

Verfaellie, M., Bowers, D., \& Heilman, K. M. (1988). Attentional factors in the occurrence of stimulus-response compatibility effects. Neuropsychologia, 26, 435-444.

Vingilis-Jaremko, L., Ferber, S., \& Pratt, J. (2008). Better late than never: how onsets and offsets influence prior entry and exit. Psychological Research, 72, 443-450.

Yantis, S., \& Jonides, J. (1996). Attentional capture by abrupt onsets: New perceptual objects or visual masking? Journal of Experimental Psychology: Human Perception and Performance, 22, 15051513.

Zimba, L. D., \& Brito, C. F. (1995). Attention precuing and Simon effects: a test of the attention-coding account of the Simon effect. Psychological Research, 58, 102-118. 